\title{
Prevalence rate of neck, shoulder and lower back pain in association with age, body mass index and gender among Malaysian office workers
}

\begin{abstract}
Background: Malaysian office workers often experience Musculoskeletal Discomfort (MSD) which is typically related to the low back, shoulders, and neck. Objectives: The objective of this study was to examine the occurrence of lower back, shoulder, and neck pain among Malaysian office workers. Methods: 752 subjects (478 women and 274 men) were randomly selected from the Malaysian office workers population of 10,000 individuals. The participants were aged between 20-50 years and had at least one year of work experience. All participants completed the Cornell Musculoskeletal Discomfort Questionnaire (CMDQ). Instructions to complete the questinnaire were given to the participants under the researchers supervision in the morning before they started a day of work. The participants were then classified into four categories based on body mas index (BMI) (BMI: $\leq 18.4,18.5-24.99,25-29.99, \geq 30)$ and age (Age: 20-29, 30-39, 40-49, $\geq 50$ ). Results: There was a significant association between pain severity in gender and right $(\mathrm{p}=0.046)$ and left $(\mathrm{p}=0.041)$ sides of the shoulders. There was also a significant association between BMI and severity of pain in the lower back area ( $\mathrm{p}=$ 0.047). It was revealed that total pain score in the shoulders was significantly associated with age $(\mathrm{p}=0.041)$. Conclusions: The results of this study demonstrated that a significant correlation existed between pain servity for gender in both right and left shoulder. These findings require further scientific investigation as do the identification of effective preventative stratgies.
\end{abstract}

Keyword: Physical pain; Musculoskeletal discomfort; Cornell questionnaire 